\title{
Redox protein Memo1 coordinates FGF23-driven signaling and small Rho-GTPases in the mouse kidney
}

\author{
Katalin Bartos $^{1,2_{\star}}$, Suresh Krishna Ramakrishnan ${ }^{2,3_{\star}}$, Sophie Braga-Lagache ${ }^{4}$, Barbara Hänzi ${ }^{3}$, \\ Fanny Durussel $^{3}$, Arjun Prakash Sridharan ${ }^{5}$, Yao Zhu ${ }^{5,6}$, David Sheehan ${ }^{5,7}$, Nancy E. Hynes ${ }^{8}$, \\ Olivier Bonny $^{2,3,9}$, Matthias B. Moor ${ }^{1,2,3}$
}

${ }^{1}$ Department of Nephrology and Hypertension, Bern University Hospital and Department of Biomedical Research, University of Bern, Bern, Switzerland

${ }^{2}$ National Center of Competence in Research (NCCR) Kidney Control of Homeostasis (Kidney.CH), University of Zurich, Zurich, Switzerland.

${ }^{3}$ Department of Biomedical Sciences, University of Lausanne, Lausanne, Switzerland

${ }^{4}$ Proteomics \& Mass Spectrometry Core Facility, Department for Biomedical Research (DBMR), University of Berne, Berne, Switzerland

${ }^{5}$ Proteomic Research Group, School of Biochemistry and Cell Biology, University College Cork, Ireland

${ }^{6}$ current address: School of Food and Nutritional Sciences, University College Cork, Cork, Ireland

7 Department of Chemistry, College of Arts and Sciences, Khalifa University of Science and Technology, Abu Dhabi, United Arab Emirates

${ }^{8}$ Friedrich Miescher Institute for Biomedical Research and University of Basel, Basel, Switzerland

${ }^{9}$ Service of Nephrology, Department of Medicine, Lausanne University Hospital, Lausanne,

Switzerland

${ }^{*}$ Co-first authors.

Running title: FGF23 responses require Memo1-dependent redox signaling

Correspondence to:

Matthias Moor, MD PhD

University Hospital Bern

Department of Nephrology and Hypertension

Freiburgstrasse 15

3010 Bern, Switzerland

matthias.moor@dbmr.unibe.ch

$+41316323144$

or

Olivier Bonny, MD PhD

University of Lausanne

Department of Biomedical Sciences

Rue du Bugnon 27

1011 Lausanne, Switzerland

olivier.bonny@unil.ch

$+41216925350$

Keywords: Memo1, FGF23, FGFR, signal transduction, RhoA, Rac1 


\section{Abstract}

Memo promotes receptor tyrosine kinase (RTK) signaling by unknown mechanisms. Memo1 deletion in mice causes premature aging and unbalanced metabolism partially resembling Fgf23 and Klotho lossof-function animals. Here, we report a role for Memo's redox function in FGF23-driven RTK signaling in the kidney. Postnatally Memo-deficient (cKO) and floxed controls were treated with FGF23 or vehicle, followed by molecular and biochemical analyses. Findings were validated using cell culture and recombinant proteins. Memo cKO mice showed impaired renal ERK phosphorylation and transcriptional responses to FGF23. Redox proteomics revealed excessive thiols of Rho-GDP dissociation inhibitor 1 (Rho-GDI1). Renal RhoA abundance and activity were increased in Memo cKO. Immunoprecipitation analysis showed an association between Memo and Rho-GDI1. We confirmed an interaction between the two proteins, with Memo-dependent irreversible oxidation at Rho-GDI1 Cys79 in cell-free conditions. Collectively, our findings reveal that redox protein Memo promotes renal FGF23 signaling together with oxidative modulation of the Rho-GTPase network. 


\section{Introduction}

Mediator of Cell Motility 1 (Memo) is an evolutionarily conserved protein associated with the cytoskeleton and receptor tyrosine kinases (1-4). In cultured cells, Memo promotes signaling and migration induced by fibroblast growth factors (FGFs) and assists in recruiting intracellular adaptor proteins to the FGF receptor $(2,5)$. In mice, postnatally-inducible ubiquitous deletion of Memo1 exon 2 (Memo cKO) resulted in a syndrome of premature aging and insulin hypersensitivity (5) which strikingly overlaps with the unusual phenotypes of Fgf23 and Klotho-deficient mouse models (6,7). Four to eight weeks after Memo1 recombination, Memo cKO animals developed kidney failure, elevated serum concentrations of FGF23, calcium and magnesium, and a bone and mineral disease $(5,8-10)$. We hypothesized that Memo modulates renal FGF23-induced cellular signaling in the kidney and in the experiments presented here, we addressed the underlying mechanisms. We demonstrate that Memo regulates activity and abundance of small Rho-GTPases and proteomic oxidation that is required for physiological intracellular signaling responses to FGF23.

\section{Results and discussion}

We generated inducible Memo cKO and floxed control mice and maintained them on C57BL/6 background as previously described (8). We treated mice of both genotypes with tamoxifen to excise Memo1 exon 2 in the whole body. We randomized mice of both genotypes to intraperitoneal FGF23 or vehicle injections as depicted in (Figure $1 \mathrm{~A}$ ) and studied all mice prior to onset of the previously described premature aging and lethal phenotype (5). Body weight (Supplemental Figure 1A), serum creatinine (Supplemental Figure 1B), calcium and phosphate (Supplemental Figure 1C-D), were comparable between genotypes, and as expected Memo protein was absent in the kidney (Supplemental Figure 1E). We assessed phosphorylated and total ERK protein in whole kidney and detected an increase in phospho-ERK in control mice treated with FGF23, but not in FGF23-treated Memo cKO animals (Figure 1B-C). Next, we analyzed expression of ERK downstream genes in kidney of mice in both genotype and treatment groups. After 1 hour of FGF23 treatment there were increases in Cyp24a1 (Figure 1D), Egr1 (Supplemental figure 1F) and cFos (Supplemental figure 1G) in control mice, but not in FGF23-treated cKO, confirming a role for Memo in FGF23-driven cell signaling in the kidney. Transcripts of FGFR regulator SEF, encoded by II17rd, were unchanged across conditions 
A

\section{Memo fl/fl control}
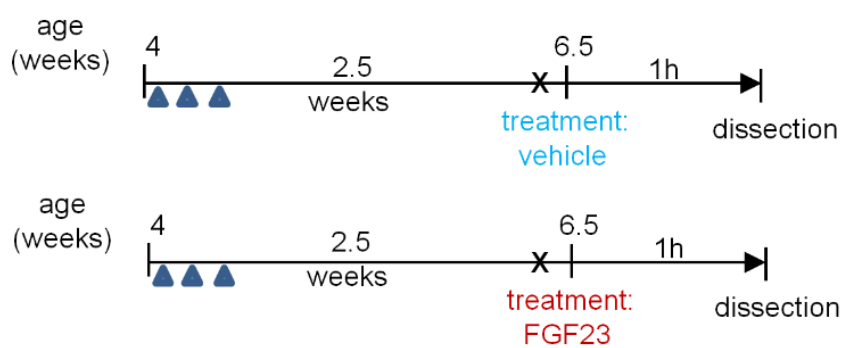

- Tamoxifen (IP 2mg) Vehicle (IP, PBS-BSA)

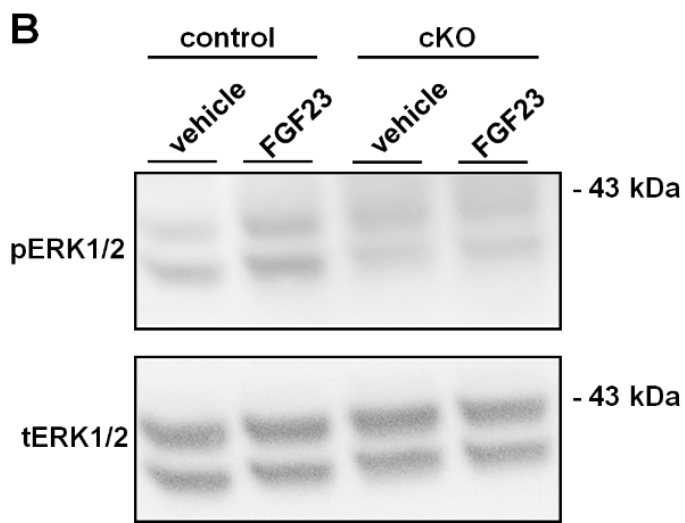

D

renal Cyp24a1 gene expression

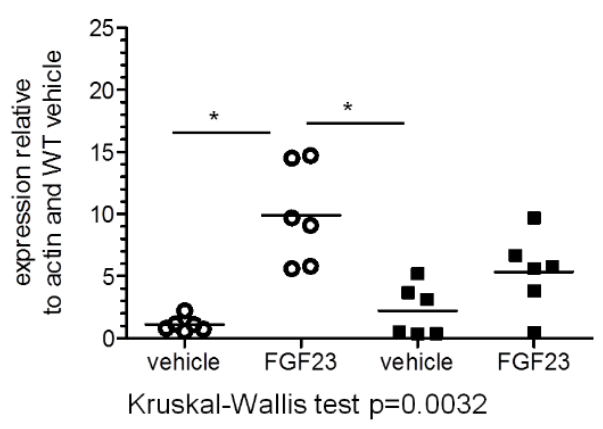

E

\section{Memo cKO}
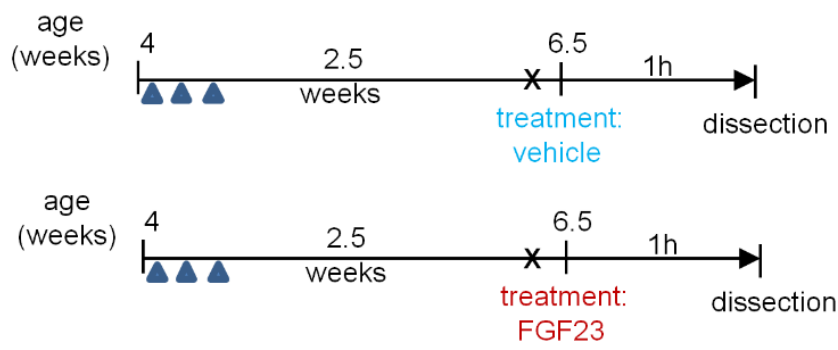

FGF23 (IP, 220ng/g)

$X$ Fasting 6 hours before FGF23 or vehicle treatment

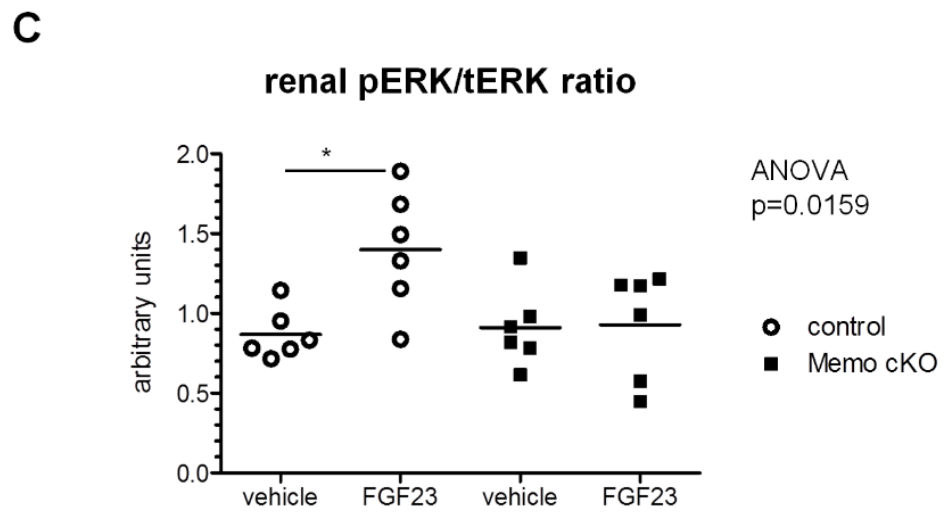

top 10 transcripts affected by treatment $x$ genotype interaction
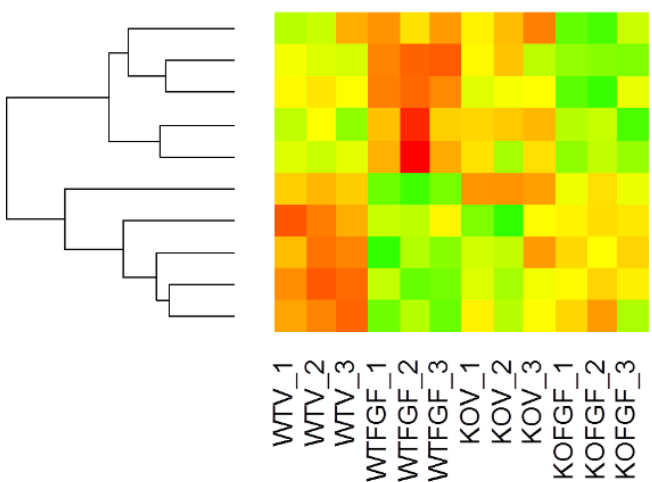

1600002H07Rik

Csrnp3

Lrrc34

P2rx1

Lrrc17

Nab2

Rcan1

Polr3d

Yrdc

Atf3

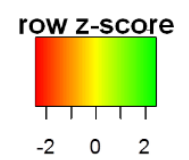

Figure 1. Impaired FGF23-induced renal signaling in whole-body Memo KO (cKO) mice. Mice were randomized to recombinant FGF23 or vehicle injection 2.5 weeks after Memo ablation (A). Phosphorylated $(p)$ versus total $(t)$ renal ERK protein assessed by Western blot 1 hour after FGF23 or vehicle (B). Densitometric quantification showed a blunted increase in $\mathrm{pERK} / \mathrm{tERK}$ ratio in response to FGF23 in kidney of Memo cKO (C). Target gene response of Cyp24a1 (D) was assessed by qPCR. Figure 1 continued on next page. 
Figure 1 continued.

Top transcripts in RNAseq affected by FGF23 treatment and genotype interaction, i.e. reacting differently to treatment across genotypes, are shown in E. Statistical analysis was obtained by ANOVA with Bonferroni's post-tests (C) or Kruskal-Wallis test with Dunn's post-test (D) between all groups, or using the heatmap2 function in R package gplots $(E) . p$ *, $p<0.05$ in post-tests. $n=6$ per condition (B-C), kidneys of 6 mice per condition pairwise pooled $(E)$.

(Supplemental figure $1 \mathrm{H}$ ), as was renal protein abundance of the FGF23 co-receptor Klotho (Supplemental figure 11).

To gain further insight into FGF23-dependent signaling pathways disturbed in Memo cKO, we assessed the transcriptome of pair-wise pooled kidney samples from FGF23-treated and vehicle-treated Memo cKO and control mice (Supplemental tables 1-5). In the acute response 1 hour after FGF23 treatment, a strong genotype effect but no overall treatment effect was visible in clustering and principal component analysis of 13362 protein-coding transcripts (Supplemental figure 2A-B). Thirteen transcripts were strongly increased by FGF23 in control genotypes but only 1 in Memo cKO (Supplemental figure 2C). Some transcripts showed a diverging FGF23 treatment effect across genotypes: Csrnp3 encoding a regulatory subunit of protein phosphatase 1 was repressed by FGF23 in control mice but increased by FGF23 in Memo cKO (Figure 1E, Supplemental figure 3A; Supplemental table 5). In contrast, a calcineurin-driven feedback regulator Rcan1 (11) was increased by FGF23 in controls but tended to decrease upon FGF23 treatment in Memo cKO (Figure 1E, Supplemental figure 3B, Supplemental table 5). This suggests that there is an FGF23-driven activation of the FGFR4 - phospholipase $\mathrm{C}_{\gamma}$ - calcineurin signaling pathway in controls, but not in Memo cKO mice. Klotho transcripts were similar in all genotypes and treatment groups (Supplemental figure 3C, Supplemental tables 3-4). Gene ontology of transcripts was analyzed (Supplemental tables $1-5,2^{\text {nd }}$ to $4^{\text {th }}$ sheet and Supplemental figures $\left.4-6\right)$. Transcripts functionally annotated to mitogen-activated protein kinase (MAPK or ERK) phosphatase activity were significantly enriched by FGF23 treatment in Memo cKO (Supplemental figure 4 and Supplemental table 1), however FGF23-treated control mice showed much stronger enrichment (Supplemental figure 5, Supplemental table 2). Moreover, in interaction analysis of genotype and FGF23 treatment this effect remained significant (Supplemental figure 6, Supplemental table 5), indicating much stronger responses to FGF23 in Memo controls on global transcriptomic levels.

We interpret the results as follows: The MAPK phosphatase-related transcripts which act as negative regulators on the MAPK cascade are quantitatively more affected by FGF23 in controls compared to in 
bioRxiv preprint doi: https://doi.org/10.1101/2020.12.04.402511; this version posted December 11,2020 . The copyright holder for this preprint (which was not certified by peer review) is the author/funder, who has granted bioRxiv a license to display the preprint in perpetuity. It is made available under aCC-BY-NC-ND 4.0 International license.

Memo cKO. However, the diverging alterations in Csrnp3 and Rcan1 (Figure 1E) rather represent a qualitative signaling alteration under Memo deletion.

Subsequently, we aimed at understanding the mechanisms of Memo by which it promotes FGF23 signaling. Memo protein itself has no phosphatase activity (12) which could influence MAPK signaling. Importantly, however, RTK-dependent signal propagation depends on redox signaling: Protein tyrosine phosphatases that constitutively control kinase cascades are regulated by cysteine oxidation in defined spatial areas of the cell $(13,14)$, showing an increase in phosphatase activity in response to low-dose oxidants but a decrease at high-dose oxidants (15). For serine and threonine phosphatases such as calcineurin or PP1, the oxidation state of a Fe(II) atom determines their activity (16). Memo structurally resembles bacterial nonheme iron dioxygenases (12) and has a copper-reducing redox function (17). Hence, we hypothesized that Memo's redox function drives FGF23-driven cellular signaling.

We used redox proteomics to determine the abundance of thiols and carbonyls as intermediates of oxidative reactions in kidney of FGF23 or vehicle-treated Memo cKO and controls. Total protein thiol content assessed by 5-iodoacetaminofluorescein (IAF) showed comparable levels between vehicletreated control and Memo cKO mice (Figure 2A). IAF signals were 2.17-fold increased in FGF23-treated controls, but 2.54-fold in FGF23-treated Memo cKO, indicating that there are differences in FGF23driven global cysteine oxidation between genotypes (Figure 2A). Total protein carbonyl content was 1.95-fold increased by FGF23 in controls, but only 1.45-fold in Memo cKO (Figure 2B), signifying that kidneys from Memo cKO animals are unable to normally process reactive intermediates (e.g. by peroxidation), which leads to uncontrolled protein thiolation.

We next determined the molecular identities of affected proteins process with altered oxidation states. We assessed the same kidney samples using 2-dimensional gel electrophoresis (2DGE) with separation by isoelectric point and mass, and used software-assisted determination of altered protein spots. Supplemental figure 7 shows 2DGE analyses of protein spots that had Coomassie, IAF or 5thiosemicarbazide (FTSC) signals which differed between controls and Memo cKO. Table 1 shows the corresponding protein identities as determined by MALDI-TOF/MS. In addition to differentially oxidized 
A

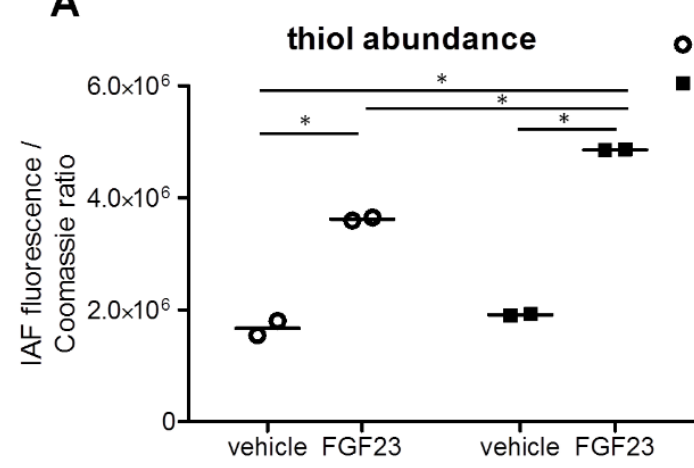

ANOVA $p<0.0001$

C

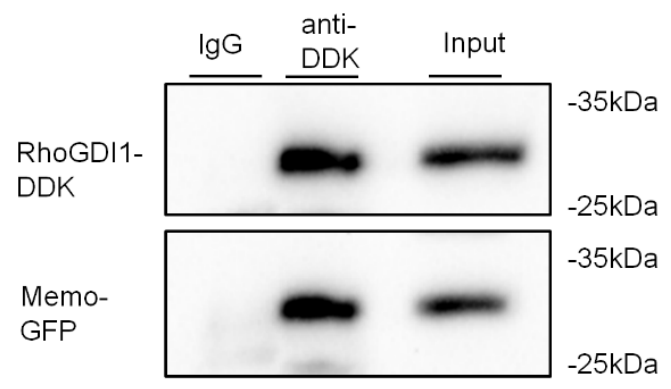

B

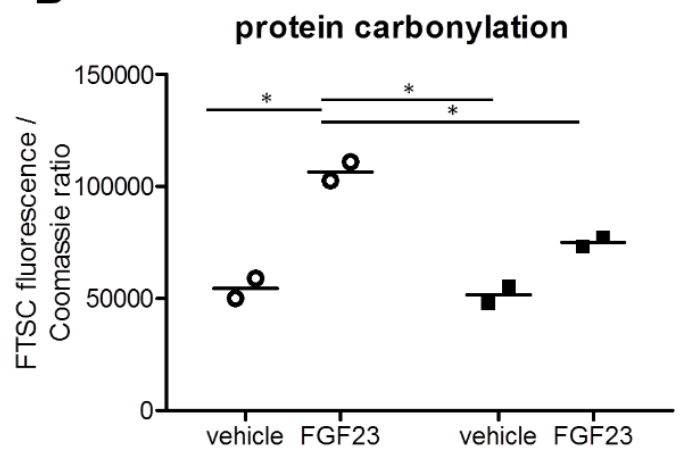

ANOVA $p=0.0015$

D

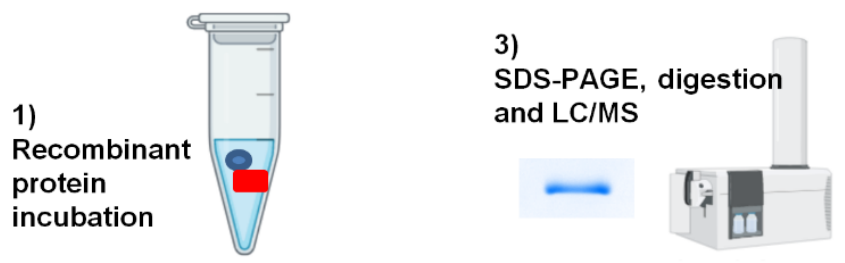
2a)

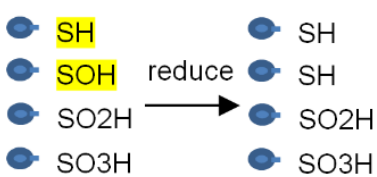

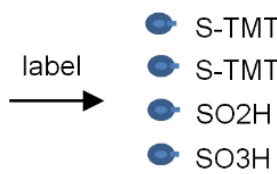

2b)

Label

Cys79

sulfenyl groups

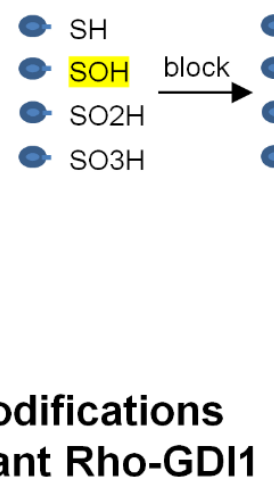

\section{posttranslational modifications
of Cys79 in recombinant Rho-GDI1}

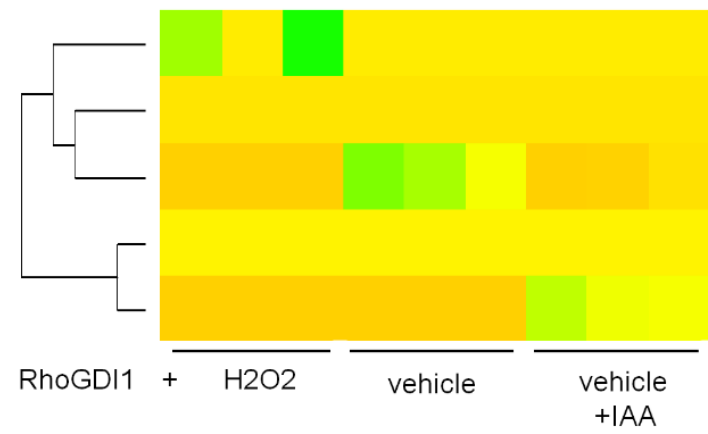

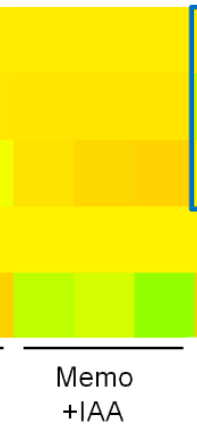
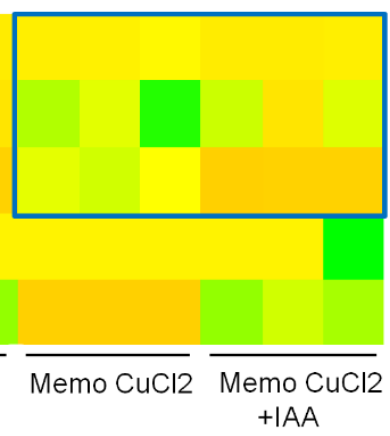

+ IAA

row z-score

Figure 2. Redox proteomic analyses and validation of interaction between MEMO and Rho-GDI1. Quantification of the redox-sensitive thiol conjugate 5-iodoacetaminofluorescein (IAF) revealed that in response to FGF23 there was a moderate 2.17-fold increase in protein thiol content FGF23 in control mice, but a 2.54-fold increase in Memo cKO (A). Figure 2 continued on next page. 
bioRxiv preprint doi: https://doi.org/10.1101/2020.12.04.402511; this version posted December 11, 2020. The copyright holder for this preprint (which was not certified by peer review) is the author/funder, who has granted bioRxiv a license to display the preprint in perpetuity. It is made available under aCC-BY-NC-ND 4.0 International license.

Figure 2 continued.

Global protein carbonylation measured by fluorescein-5-thiosemicarbazide (FTSC) was increased 1.95fold by FGF23 in controls, but only 1.45 -fold in Memo cKO (B). Each data point represents means of 3 technical replicates of pooled kidney halves of 2 animals $(n=2 \times 2$ per condition). Measured intensities were normalized by Coomassie stained total protein. Panel C shows lysates of HEK293 cells transfected with DDK-Rho-GDI1 and GFP-Memo vectors that were immunoprecipitated with a DDK antiserum and probed for DDK-Rho-GDI1 and GFP-MEMO; representative of three independent experiments. Panel D shows the experimental workflow for modification analyses of Cys79 in recombinant Rho-GDI1, after incubation with or without copper pre-loaded Memo or H2O2. In brief, irreversibly dioxidized (SO2H) and trioxidized $(\mathrm{SO} 3 \mathrm{H})$ were directly detected by MALDI-TOF/MS, and reversible monooxidation $(\mathrm{SOH})$ peptides were detected using a iodoTMT tandem mass-spectroscopy tag (TMT) after blocking accessible cysteines by iodoacetamide (IAA); total accessible cysteines were detected without IAA blocking. Incubation of RhoGDI1 with $\mathrm{H} 2 \mathrm{O} 2$ as a positive control caused trioxidation (E, first row), and incubation with copper-preloaded and dialyzed Memo caused dioxidation and to a lesser extent trioxidation in RhoGDI1 Cys79 (E, blue rectangle). Memo oder copper-preloaded Memo addition did not alter the TMT label intensity (E, $3^{\text {rd }}$ row and blue rectangle). Only one sample had some remaining free thiols $\left(E, 4^{\text {th }}\right.$ row), and in all IAA-blocked samples, the IAA alkylation was present (E, $5^{\text {th }}$ row). Signal intensities in $\mathrm{E}$ are normalized for total number intensity of detected peptides, and displayed as Z-scores of original non-transformed data. Statistical analysis was obtained by ANOVA with Bonferroni's multiple testing correction performed between all groups (A-B) or Kruskal-Wallis test with Dunn's multiple testing correction (E). ${ }^{*}, p<0.05$ in post-tests. $n=2 \times 2$ animals pooled per condition $(A, B) ; n=3$ independent experiments per condition $(C, E)$.

proteins associated with cytoskeleton and carbohydrate metabolism, we found one potential signalingrelated protein, the Rho-GDP dissociation inhibitor 1 (Rho-GDI1), a chaperone protein of Rho-GTPases. The renal thiols of Rho-GDI1 were 1.69-fold increased in FGF23-treated Memo cKO compared to controls (Table 1$)$.

We experimentally tested the possibility that Memo and Rho-GDI1 interact using two approaches: First, lysates from HEK293 cells transfected with vectors expressing GFP-tagged Memo1 and DDK-tagged Rho-GDI1 were immunoprecipitated with anti-DDK antibody, then probed for Memo-GFP. The results show a co-immunoprecipitation of both proteins (Figure 2C). Next, we determined if recombinant Memo can functionally affect Rho-GDI1 by oxidation of its single cysteine at position 79 . We quantified posttranslational modifications of Rho-GDI1 Cys79 in native peptides and using sulfhydryl-specific tandem mass tag (TMT) iodoTMT in a cell-free environment (Figure 2D). After pre-loading Memo protein with $\mathrm{CuCl}_{2}$ as the putative cofactor copper, followed by dialysis to remove of free copper, the reversible oxidation of Rho-GDI1 Cys79 to sulfenate was negligible, but we detected irreversible dioxidation to sulfinyl groups $(\mathrm{SO} 2 \mathrm{H})$ and to some extent trioxidation to sulfonyl groups $(\mathrm{SO} H)$ at Cys79 of Rho-GDI1 (Figure 2E). H2O2 served as a positive control. These findings support the data from the 2DGE screen (Table 1) and reveal that the effects on Rho-GDI1 are similar to the previously reported effect of Memo on RhoA, its interaction partner RhoA. In this case, RhoA displayed reduced cysteines in Memo-deficient cells, but was directly oxidized by recombinant Memo (17). MacDonald et al. also demonstrated an increase of Memo oxidase activity upon copper loading (17), similar as the current findings of irreversible 
bioRxiv preprint doi: https://doi.org/10.1101/2020.12.04.402511; this version posted December 11, 2020. The copyright holder for this preprint (which was not certified by peer review) is the author/funder, who has granted bioRxiv a license to display the preprint in perpetuity. It is made available under aCC-BY-NC-ND 4.0 International license.

Cys79 oxidation. Overall, the interaction between Memo and Rho-GTPases results in differential regulation of multiprotein subunit recruitment of NADPH oxidases $(17,18)$, and the current data provide

Table 1: Identification of altered proteins in redox proteomics of the kidney

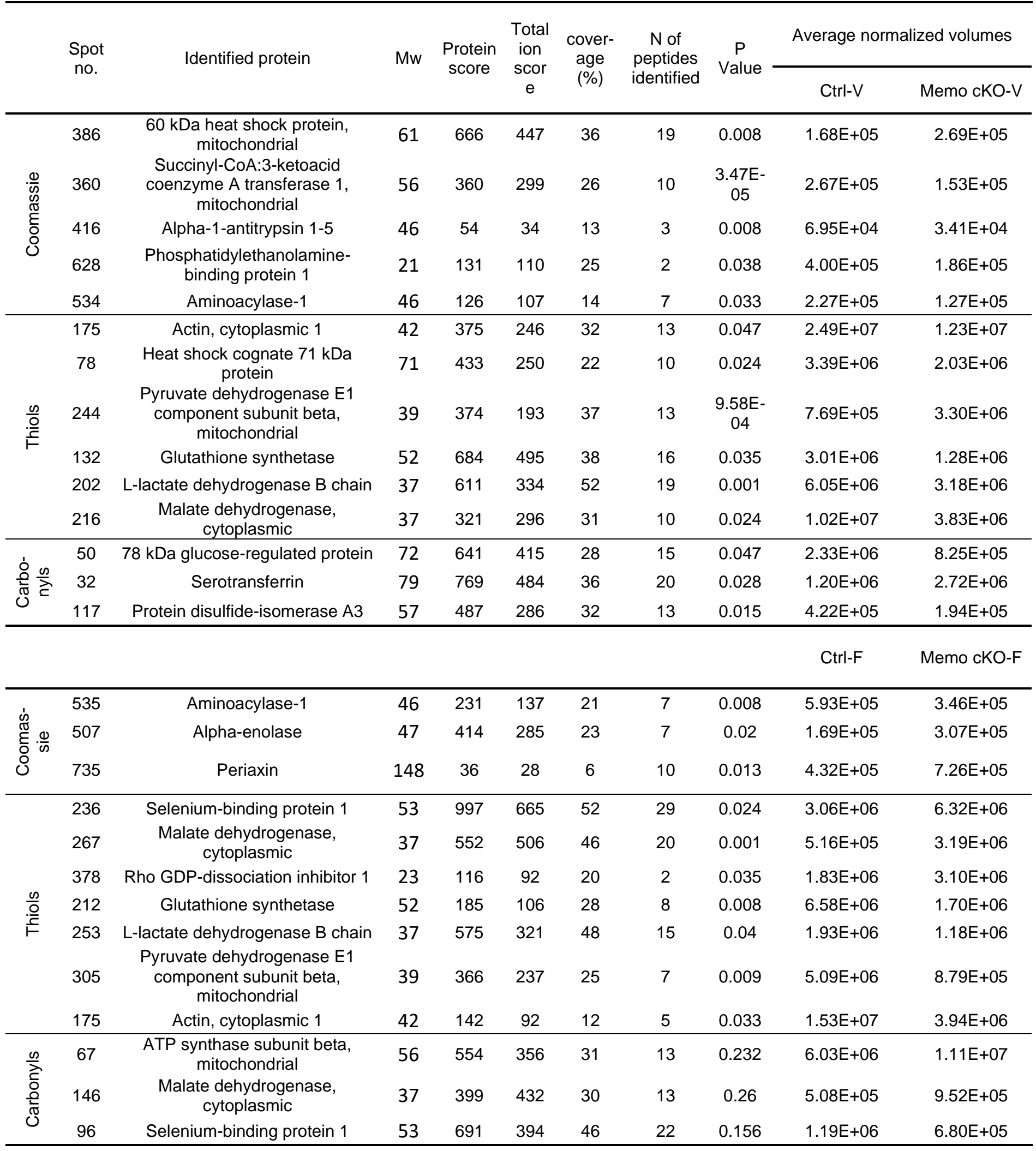

Mw, molecular weight in kDa. Ctrl, control. V, vehicle. F, FGF23. P-Values determined by t-test. 
a novel link via Rho-GDI1 which has a known role in kidney diseases $(19,20)$. Moreover, results from cysteine oxidation proteomics have recently highlighted extensive alterations in the oxidation of RhoGTPase subfamilies RHO and RAC in response to epidermal growth factor-driven RTK signaling (21).

Protein abundance of Rho-GDI1 was comparable between genotypes and treatment groups (Figure 3A, quantification in Figure 3E). With total Rho-GDI1 unchanged, the less abundant Rho-GDI1 thiols in FGF23-treated control mice would correspond to transient oxidation of Rho-GDI1 Cys79 to sulfenate in order to liberate and activate a bound Rho-GTPase. This has previously been demonstrated for the RhoA - Rho-GDI1 complex (22). Memo is required for growth factor-driven RhoA relocalization to the membrane (1), and strikingly, RhoA protein abundance and activity were increased in the kidney of Memo cKO mice (Figure 3B, 3E-3F). The abundance of Rho-GTPase Rac1 was unchanged in all conditions (Figure 3C, quantification in Figure 3E). However, FGF23 treatment caused a trend to $10 \%$ increase in Rac1 activity in kidney of control mice (Figure 3F), similar to the previously reported effect of FGF2 treatment in cell culture $(23,24)$. In the kidney of Memo cKO animals, however, this trend was absent.

These findings suggest that the Rho-GTPases network is disturbed in the absence of Memo, which has implications for the well-characterized intracellular redox signaling by Rac1 leading to NADPH oxidase assembly (25) and for RTK endocytosis which is regulated by both RhoA and Rac1 and is a cue to stop FGFR-mediated MAPK signaling (26-28). Intriguingly, Snap91 was the top upregulated transcript (Supplemental figure 8) in FGF23-treated Memo cKO compared to FGF23-treated controls. Snap91 encodes a clathrin coat assembly protein that aids in recognizing membrane receptor cargo to drive endocytosis (29). Collectively, this raises exciting questions about the potential of Memo at the intersection of RTK signaling, redox biology and membrane trafficking of RTK.

To conclude, here we present results that shed light on the role of Memo in response to FGF23-driven RTK signaling in the kidney. Our data provide insight into the in vivo physiological role and the underlying 
A

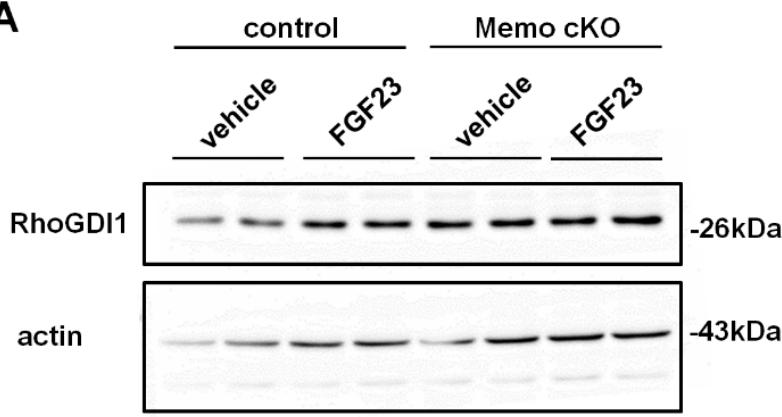

C

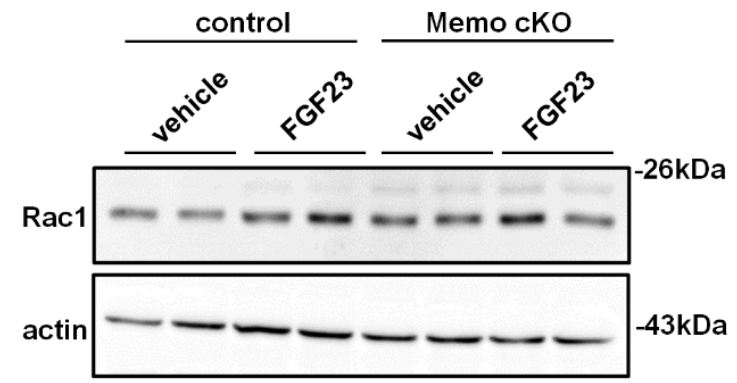

B

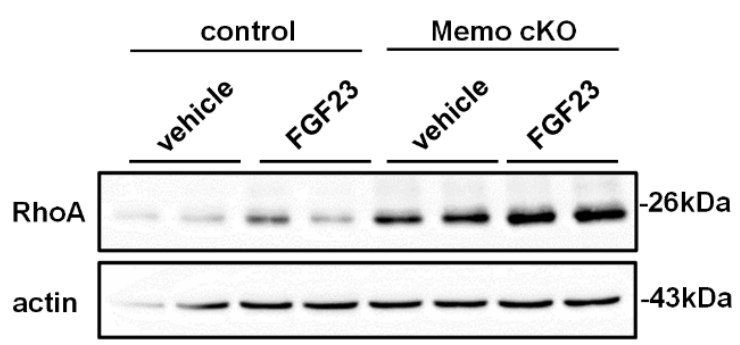

D

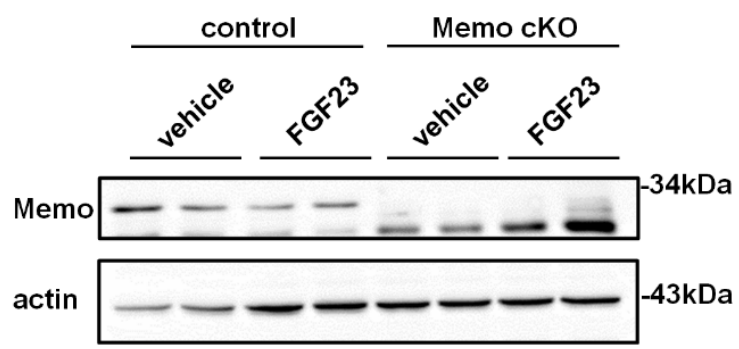

$\mathbf{E}$

\section{renal protein abundance}

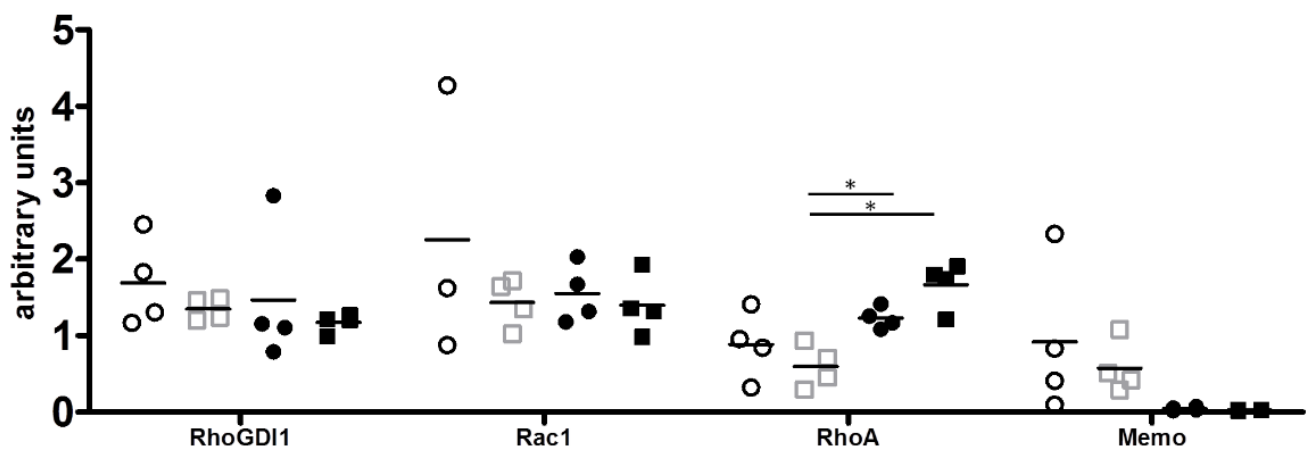

- control - vehicle

$\square$ control - FGF23

- Memo cKO - vehicle

- Memo cKO - FGF23

ANOVA

RhoGDI1 $\quad p=0.6152$

Rac1

RhoA

Memo

=0.5396

$p=0.0025$

$p=0.0891$

$\mathbf{F}$

renal small GTPase activity

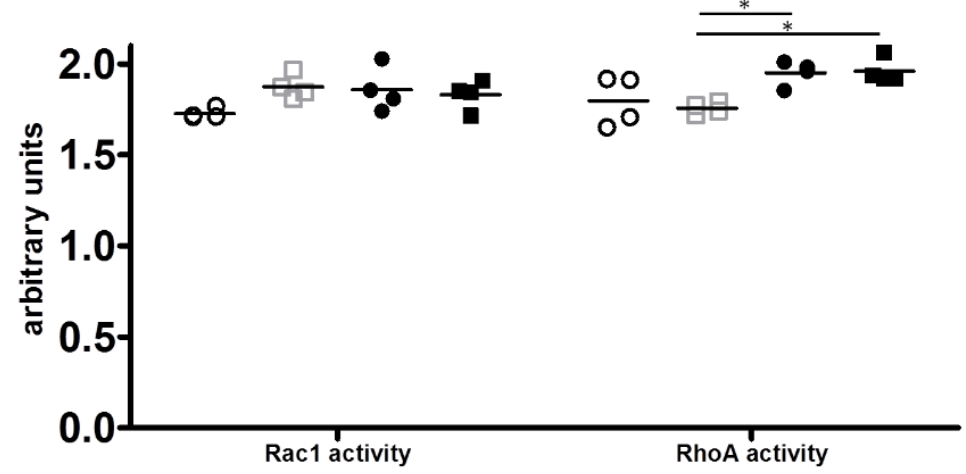

- control-vehicle

control - FGF23

- Memo cKO - vehicle

- Memo cKO - FGF23

ANOVA:

Rac1 $\quad p=0.0989$

RhoA $\quad p=0.0102$

Figure 3. The Rho-GTPase network in kidneys from whole-body Memo cKO (cKO) mice is malfunctioning. Western blotting of renal lysates shows unchanged levels of Rho-GDI1 (A) and Rac1 (C) in Memo cKO compared to control mice; RhoA (B) was increased in Memo cKO mice. Protein band at $33 \mathrm{kDa}$, specific for Memo protein, was absent in kidney from Memo cKO (D). Densitometric quantifications of Western blots A-D, relative to actin are shown in E. Renal Rac1 activity showed a trend to increase in control mice treated with FGF23, but was not significantly changed (ANOVA $\mathrm{p}=0.099)$. ( $\mathrm{F}$, left panel). Figure 3 continued on next page. 
Figure 3 continued.

Renal RhoA activity was significantly higher in Memo cKO (F, right panel). Statistical analyses in E-F were performed by ANOVA with Bonferroni correction for multiple testing (6 post-tests each). *, posttest $p<0.05$. $n=4$ per condition in $A-F . n=4$ per condition $(A-F)$; except $n=3$ in $E$ (Rac1: control vehicle) due to undetectable loading control.

mechanisms how the evolutionarily conserved moonlighting protein Memo simultaneously modulates multiple RTK-dependent signaling pathways.

\section{Methods}

\section{Animal studies}

All animal experimental protocols were approved by the veterinary service of the Canton de Vaud, Switzerland. Mice were fed standard chow (TS3242 Kliba Nafnag, Kaiseraugst, Switzerland) and were kept on 12/12 or 14/10 light-dark cycles. Mice floxed for exon 2 of the Memo1 gene (5) backcrossed to C57BL/6J background over 10 generations were crossed with CreERTM transgenic mice (30) carrying a tamoxifen-inducible Cre recombinase controlled by a beta-actin promoter/enhancer. Genotypes were determined by PCR of ear punch biopsy DNA using primers: Memo forward 5'CCCTCTCATCTGGCTTGGTA-3', Memo reverse 5'- GCTGCATATGCTCACAAAGG-3', Cre forward 5'AGGTTCGTGCACTCATGGA-3', Cre reverse 5'-TCACCAGTTTAGTTACCC-3'. For the current study, all animals used were males, with the exception of the experiments reported in Figure 3 in which there was a sex ratio of 50:50 in each genotype. Loss of Memo was induced by 3 daily intraperitoneal injections with $2 \mathrm{mg}$ tamoxifen at age 4 weeks (T5648 Sigma-Aldrich, distributed through Merck, Buchs Switzerland). Memofl/1/1 littermates without Cre but treated with tamoxifen served as controls. For FGF23 treatments, mice aged 6.5 weeks were starved for 6 hours and intraperitoneally injected with $220 \mathrm{ng} / \mathrm{g}$ body weight of recombinant mouse FGF23 (2629-FG-025 R\&D Systems, Minneapolis, MN, USA) in PBS-BSA $0.1 \%$ or PBS-BSA $0.1 \%$ vehicle with injection volume of $4 \mu \mathrm{L} / \mathrm{g}$ body weight. One hour later mice were dissected, a protocol adapted from (31). Treatments were randomly allocated by flipping a coin. Animals were euthanized after $1 \mathrm{~h}$ by terminal exsanguination under anesthesia.

\section{Transfection}

Hek293 cells were obtained from ATCC and were tested negative for mycoplasma using LookOut $\circledast$ PCR-based kit (MP0035-1KT Sigma-Aldrich). Cells were transfected with GFP-tagged Memo1 (MG204118 OriGene Technologies Inc., Rockville MD, USA) and Myc-DDK-tagged Rho-GDI1 
bioRxiv preprint doi: https://doi.org/10.1101/2020.12.04.402511; this version posted December $11,2020$. The copyright holder for this preprint (which was not certified by peer review) is the author/funder, who has granted bioRxiv a license to display the preprint in perpetuity. It is made available under aCC-BY-NC-ND 4.0 International license.

(ARHGDIA) (MR202112 OriGene) using Lipofectamine 3000 (L3000015 Invitrogen, distributed through Thermo Fisher Scientific, Reinach, Switzerland).

\section{Redox proteomics}

Kidney halves pooled from 2 mice per sample were homogenized in $10 \mathrm{mM}$ Tris- $\mathrm{HCl}, \mathrm{pH}$ 7.2, $250 \mathrm{mM}$ sucrose, $1 \mathrm{mM}$ EDTA, $150 \mathrm{mM} \mathrm{KCl}$ and $1 \mathrm{mM} \mathrm{PMSF}$ and spun down. Protein thiols and carbonyls were labelled with either $0.2 \mathrm{mM} \mathrm{5'-iodoacetamido} \mathrm{fluorescein} \mathrm{(IAF)} \mathrm{or} 1 \mathrm{mM}$ fluorescein-5-thiosemicarbazide (FTSC), respectively, and incubated for $150 \mathrm{~min}$ at $37^{\circ} \mathrm{C}$ in the dark. Proteins were precipitated with 20 $\%$ TCA and centrifuged at $20,000 \times \mathrm{g}\left(3 \mathrm{~min}, 4^{\circ} \mathrm{C}\right)$. Protein pellets were resuspended and washed with $100 \%$ ethanol/ethyl acetate $(1: 1)$ and $96 \%$ acetone, respectively, for carbonyl and thiol groups. Pellets were resuspended in Tris- $\mathrm{HCl} 0.5 \mathrm{M} \mathrm{pH} 6.8$, glycerol 10\%, SDS $0.5 \%$ and bromophenol blue and applied to SDS-PAGE. Gels were scanned for fluorescence in a Typhoon Trio Scanner 9400 (Control v5.0 + variable Mode Imager-RA 501: PRT<I/06/004, GE Healthcare, Buckingshamshire, UK; excitation, 490-495 nm; emission, 515-520 nm). Protein-associated fluorescence intensities (arbitrary units, AU) were analyzed using Quantity One image analysis software (BioRad, Hercules, CA, USA). Gels were stained with Colloidal Coomassie Brilliant Blue G250 and scanned.

For two dimensional analysis, proteins were first separated by pl (first dimension: isoelectric focusing IEF), followed by orthogonal separation according to molecular weight (second dimension: SDS-PAGE). Proteins were rehydrated in $5 \mathrm{M}$ urea, $2 \mathrm{M}$ thiourea, 2\% CHAPS, 4\% ampholyte (Pharmalyte 3-10, Amersham-Pharmacia Biotech, Little Chalfont, Bucks, UK), 1\% Destreak reagent (AmershamPharmacia Biotech, Buckinghamshire, UK), and trace amounts of bromophenol blue, and then immobilized in $7 \mathrm{~cm}$ IPG strips $(\mathrm{pH} 3-10: 70 \times 3 \times 0.5 \mathrm{~mm}$ ) and a linear gradient (NL) (GE Healthcare Immobiline ${ }^{\mathrm{TM}}$ Dry Strip IPG, GE17-6001-11 Bio-Sciences AB, Bio-Rad, Hercules, CA, USA). Proteins were focused at room temperature in a Protean IEF Cell (Bio-Rad) for at least $15 \mathrm{~h}$, according to the following steps: (1) a linear voltage increase until $250 \mathrm{~V}$ for $15 \mathrm{~min}$, (2) 10,000 V for $2 \mathrm{~h}(50 \mu \mathrm{A} / \mathrm{strip})$, (3) focusing at $20,000 \mathrm{~V}$, and (4) hold at $500 \mathrm{~V}$. Following IEF, strips were equilibrated for $20 \mathrm{~min}$ in equilibration buffer ( $6 \mathrm{M}$ urea, $0.375 \mathrm{M}$ Tris, $\mathrm{pH} 8.8,2 \% \mathrm{SDS}, 20 \%$ glycerol, containing $2 \% \mathrm{DTT}$ ) and then for 20 min in equilibration buffer containing 2.5\% iodoacetamide. IPG strips were loaded onto $12 \%$ SDS-PAGE gels (PROTEAN Plus Dodeca Cell, BioRad). Gels were scanned for fluorescence as above and stained with Coomassie Blue followed by densitometry. Progenesis SameSpots Software (S/No.62605/3787; Nonlinear USA Inc, Durham, NC USA) was used to normalize FTSC/ IAF-labeled 
protein spots and Coomassie-staining intensity. Fluorescence spots were normalized to protein intensity for the same gel.

All experiments were performed in triplicates. Images of 2D gels were subjected to landmarking alignment so that corresponding spots were matched with each other, based on 3D Gaussian distribution after raw image correction and background subtraction. Spot intensities were normalized. Differences between protein spots in 2D gel images were automatically determined.

Spots with differences of $p<0.05$ by ANOVA were manually excised from colloidal Coomassie-stained 2-DE gels. Proteins were extracted and enzymatically digested using a Perkin Elmer - Janus automated workstation. Following digestion, samples were reconstituted in $0.1 \%$ formic acid and analyzed using a Dionex U3000 Liquid Chromatography System (Dionex, Sunnyvale, CA, USA) and the Daltonics HCT Ion Trap Mass Spectrometer (Bruker, Glasgow, UK). The peptide fragment mass spectra were acquired in data-dependent AutoMS(2) mode with a scan range of $300-1500 \mathrm{~m} / \mathrm{z}$, and up to three precursor ions were selected from the MS scan $(100-2200 \mathrm{~m} / \mathrm{z})$. Precursors were actively excluded within a 1.0-min window, and all singly charged ions were excluded. Following LC-MS/MS, Mascot Generic Files were created and the MASCOT database NCBInr was searched using Matrix Science webserver (www.matrixscience.com). The default search parameters were: enzyme = trypsin; maximum number of missed cleavages $=1$; fixed modifications $=$ carbamidomethyl $(C)$; variable modifications $=$ oxidation $(\mathrm{M})$; peptide tolerance $\pm 1.5 \mathrm{Da}$; MS/MS tolerance $\pm 0.5 \mathrm{Da}$; peptide charge $=2+$ and $3+$. Identified proteins were considered if a MASCOT score above $95 \%$ confidence was obtained $(p<0.05)$ and at least one peptide was identified with a score above 95\% confidence $(p<0.05)$. This analysis was conducted at the Proteomics Core Facility of the University of Aberdeen, UK. All mass spectrometry proteomics data from these experiments have been deposited to the ProteomeXchange Consortium via the MassIVE repository with the dataset identifier PXD022342.

\section{Cysteine oxidation analyses with recombinant protein}

Amino acids 24-204 of human Rho-GDI1/ARHGDIA protein expressed in E. coli was obtained from Novusbio (NBP1-50861, Centennial, CO, USA NBP1-50861), with $>95 \%$ purity by SDS-PAGE and supplied in $20 \mathrm{mM}$ Tris-HCl buffer ( $\mathrm{pH} 8.0$ ), $1 \mathrm{mM}$ DTT, $10 \%$ glycerol without preservatives. Recombinant human Memo expressed in E. coli with $>95 \%$ purity, supplied in $20 \mathrm{mM}$ Tris-HCl buffer, $\mathrm{pH} 8.0,50 \%$ glycerol, 5 mM DTT, 300 mM NaCl, 2 mM EDTA was from Antibodies-online (ABIN2130536, Aachen, 
Germany). Copper-reducing oxidant activity of the batch of recombinant Memo protein used in this experiment was determined and reported in (9).

First, as a putative metal cofactor pre-loading step, $2 \mu \mathrm{g}$ Memo was incubated with RCM buffer with or without $10 \mu \mathrm{M} \mathrm{CuCl}_{2}$. After 30 minutes incubation at $4^{\circ} \mathrm{C}$ the free $\mathrm{CuCl}_{2}$ was removed by Slide-A-Lyzer ${ }^{\mathrm{TM}}$ MINI Dialysis Device, 3.5K MWCO (69550 Thermo Fisher Scientific) against RCM buffer for 30 minutes at $4^{\circ} \mathrm{C}$. Proteins were incubated for 15 minutes as 1000ng Rho-GDI1 alone; 1000ng Rho-GDI1 with 250ng Memo; 1000ng Rho-GDI1 with 250ng $\mathrm{CuCl}_{2}$ pretreated Memo; and 1000ng Rho-GDI1 with $100 \mu \mathrm{M}$ of hydrogen peroxide as a positive control (H1009 Sigma-Aldrich), all in duplicates for each experiment, to individually label the total cysteine and the oxidized cysteine content of Rho-GDI1.

The samples for total cysteine content labeling were reduced with $5 \mathrm{mM}$ Tris (2-carboxyethyl) phosphine (TCEP) (646547 Sigma-Aldrich). Total cysteine content was labeled with iodoTMTsixplex ${ }^{\text {TM }}$ (90102 Thermo Fisher Scientific) followed by quenching with 20mM 1DTT (43816 Sigma-Aldrich).

The samples for oxidized cysteine content labeling were treated with $500 \mathrm{mM}$ iodoacetamide (I6125 Sigma-Aldrich) for 30 minutes followed by quenching with $20 \mathrm{mM}$ DTT and reducing with $5 \mathrm{mM}$ TCEP. The oxidized cysteine content of the samples was labeled with iodoTMTsixplex ${ }^{\mathrm{TM}}$.

Samples were precipitated with the SDS-PAGE Clean-Up Kit (10074304 GE Healthcare Life Sciences, distributed through Thermo Fisher Scientific) and separated on SDS-PAGE, followed by Coomassie Blue staining and manual band excision. Proteins were digested by chymotrypsin at $50^{\circ} \mathrm{C}$ for 5 hours and analyzed by LC-MS/MS (PROXEON coupled to a QExactive HF mass spectrometer, Thermo Fisher Scientific) with two injections of $5 \mu$ l digests. Peptides were trapped on a $\mu$ Precolumn C18 PepMap100 $(5 \mu \mathrm{m}, 100 \AA, 300 \mu \mathrm{m} \times 5 \mathrm{~mm}$, Thermo Fisher Scientific) and separated by backflush on a C18 column

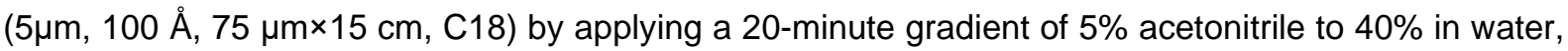
$0.1 \%$ formic acid, at a flow rate of $350 \mathrm{nl} / \mathrm{min}$. The Full Scan method was set with resolution at 60,000 with an automatic gain control (AGC) target of $1 \mathrm{E} 06$ and maximum ion injection time of 50ms. The datadependent method for precursor ion fragmentation was applied with the following settings: resolution 15,000 , AGC of $1 \mathrm{E} 05$, maximum ion time of 110 milliseconds, mass window $1.6 \mathrm{~m} / \mathrm{z}$, first mass $100 \mathrm{~m} / \mathrm{z}$, collision energy 27 , under fill ratio $1 \%$, charge exclusion of unassigned and $1+$ ions, and peptide match preferred, respectively. Spectra were interpreted with Proteome Discoverer 2.4.0.305, with chymotrypsin rules allowing up to 8 missed cleavages, using variable modification of carboamidomethylated (+57.021Da), dioxidation (+31.990Da), trioxidation $(+47.985 \mathrm{Da})$ and iodoTMT labelling $(+324.216 \mathrm{Da})$ on Cys, and variable modification of oxidation $(+15.99 \mathrm{Da})$ on Met, and 
acetylation $(+42.011 \mathrm{Da})$ on protein $\mathrm{N}-$ Term. Parent and fragment mass tolerances were set to 10ppm and $0.02 \mathrm{Da}$, respectively. Strict target false discovery rate for highly confident peptide-spectrum matches was set to 0.01 . Protein identifications were only accepted when two unique peptides fulfilling the $1 \%$ FDR criterium were identified. This analysis was performed at the Proteomics Mass Spectrometry Core Facility of University of Bern. Resulting intensity peaks of specific peptide modifications were normalized by total signal intensity and are displayed as heatmap of non-transformed z-scores, i.e. a subtraction of the row mean followed by a division by the row standard deviation. All mass spectrometry proteomics data from recombinant protein analyses have been deposited to the ProteomeXchange Consortium via the PRIDE repository with the dataset identifier PXD022382.

\section{Immunoprecipitation}

For immunoprecipitation Protein G Sepharose beads (P3296 Millipore, distributed through Merck, Buchs Switzerland) were blocked with 1\% BSA (A9647 Sigma-Aldrich) and incubated with anti-DDK Monoclonal Antibody (TA50011-100 OriGene) and Mouse IgG2a Isotype Control (02-6200 Invitrogen, distributed through Thermo Fisher Scientific) in 1:200 dilution for 45 minutes at $4^{\circ} \mathrm{C}$. After washing with RIPA (R0278 Sigma-Aldrich), the cell lysate was added to the beads in 5:1 dilution and incubated overnight. Beads were resuspended in RIPA and 4x SDS Sample buffer, heated up to $95^{\circ} \mathrm{C}$ and spun down.

\section{Western blot}

For immunoblotting, immunoprecipitates or proteins of cells and tissues were lyzed in RIPA buffer or NP-40 buffer (150mM NaCl, 50 mM HEPES pH7.4, 25mM NaF, 5mM EGTA, 1mM EDTA, 1\% Nonidet $\mathrm{P}-40,2 \mathrm{M}$ Na ortho-vandate and $1 \mathrm{mM}$ DTT supplied with protease inhibitors leupeptin $10 \mu \mathrm{g} / \mathrm{L}$, aprotinin $10 \mu \mathrm{g} / \mathrm{L}$, and PMSF $1 \mathrm{mM}$ ) were prepared and denatured. Proteins were separated by SDS-PAGE and transferred onto nitrocellulose or PVDF, stained by Ponceau S, blocked in dried nonfat milk 5\%-TBST or bovine serum albumin (A9647 Sigma-Aldrich) 3\% in TBST before incubation with primary antibodies against Memo (1:2000, produced in-house (5) or 1:1000, HPA042603 Sigma-Aldrich), GFP (1:1000, G1544 Sigma-Aldrich), or FLAG (1:1000, F7425 Millipore), pERK (1:1000, sc-7383 Santa Cruz, Dallas TX, USA), tERK (1:1000, sc-93 Santa Cruz), actin (1:2000, A2066 Sigma-Aldrich), Rho-GDI1 (1:500, ABIN969501 Antibodies-online), Rac1 (1:500, ab33186 Abcam, Cambridge UK), RhoA (1:500, NBP222529 NovusBio) and Klotho (1:1000, AF1819 R\&D Systems). Membranes were incubated with anti- 
mouse or anti-rabbit horseradish peroxidase-conjugated secondary antibodies (Milian Analytica, Rheinfelden Switzerland or ImmunoResearch, distributed through LubioScience GmbH, Zürich, Switzerland) and imaged using Fusion Solo (Witec AG, Sursee Switzerland) or a ChemiDoc XRS+ System (BioRad). For reprobing, membranes were stripped using a low pH buffer $(25 \mathrm{mM}$ glycine- $\mathrm{HCl}$, $\mathrm{pH} 2,0.4 \%(\mathrm{w} / \mathrm{v}) \mathrm{SDS})$.

\section{Biochemical analyses}

Serum electrolytes were analyzed by the Lausanne University Hospital: Total calcium (NM-BAPTA method), phosphate (phosphomolybdate method), and creatinine (modified Jaffé method).

\section{Rho-GTPase activity assays}

For quantification of Rho-GTPase activity in kidney homogenates, colorimetric Rac1 and RhoA G-LISA Activation Assay Kits (BK128-S and BK124-S Cytoskeleton Inc., Denver CO, USA) were used according to the manufacturer's instructions.

\section{RNA isolation}

Frozen kidneys were homogenized with metal beads in a TissueLyser (Qiagen, Hombrechtikon, Switzerland). RNA was extracted using TRI Reagent Solution (AM9738 Ambion, Austin, TX, USA) for all downstream applications.

\section{qPCR}

One $\mu \mathrm{g}$ of RNA per kidney half was reverse-transcribed using PrimeScript RT Reagent kit (RR037 TAKARA, Shiga, Japan). Two $\mu$ l of cDNA was used for quantitative real-time PCR to assess gene mRNA expression. Assays were performed using SYBR Green (Applied Biosystems, Foster City, CA UA) on a 7500 Fast machine (Applied Biosystems). Samples were run in triplicates in 20uL total volume for each gene, and actin or GAPDH was used for normalisation. Melting curves were obtained for every run. Program settings were: $95^{\circ} \mathrm{C} 20$ seconds, 40 cycles $\left(95^{\circ} \mathrm{C} 3 \mathrm{sec}, 60^{\circ} \mathrm{C} 30 \mathrm{sec}\right)$, and for melting curve stage: $95^{\circ} \mathrm{C} 15 \mathrm{sec}, 60^{\circ} \mathrm{C} 1 \mathrm{~min}$, rising at $1 \%$ ramp speed to $95^{\circ} \mathrm{C}(15 \mathrm{sec}), 60^{\circ} \mathrm{C} 15 \mathrm{sec}$. Data were analyzed using the delta-delta CT method. Primers were ordered from Microsynth (Balgach, Switzerland), and sequences are shown in Supplemental table 6. 


\section{RNAseq}

For RNAseq, extracted RNA from mouse kidney halves was pairwise pooled to 3 individual pools for each of 4 experimental conditions (2 treatment states, 2 genotypes). Quality of mRNA was assessed and verified using a Fragment Analyzer (Advanced Analytical Technologies Inc.). Following quality validation, TruSeq Stranded RNA Sample Prep Kit (20020596 Illumina, San Diego CA, USA) was used with 1ug of total RNA for the construction of sequencing libraries. cDNA was sequenced using the Illumina HiSeq2500 platform and single-end reads (1x100). All sequencing data are accessible on National Center for Biotechnology Information, Sequence Reads Archive, Accession: PRJNA672305. Purity-filtered reads were trimmed with Cutadapt (v. 1.3, Martin 2011), filtered using seq_crumbs (v. 0.1.8) and aligned against the Mus musculus (Ensembl version GRCh38.82) transcriptome using STAR (v. 2.4.0f1). Data were normalized with the Trimmed Means of M-Values (TMM) method of the edgeR, and transformed with voom method of bioconductor package limma. Genes expressed at low levels were removed when none of the samples had more than 1 count per million reads. Only genes labeled as "protein coding" were retained for the statistical tests. A heatmap was constructed using the heatmap2 function in R 4.0.0 package gplots using z-scores of log-transformed normalized reads.

\section{Statistical analyses}

Analyses of parametric continuous variables were performed by t-test or by ANOVA, followed by posttests using Bonferroni correction for multiple testing across all experimental groups. Analyses of nonparametric continuous variables were assessed by Kruskal Wallis test, followed by Dunn's correction for multiple testing across all experimental groups. Analyses were performed in GraphPad Prism 5.0, and two-tailed $p$-values of $<0.05$ were considered significant.

Analyses of RNAseq data were performed with limma (R version 3.2.1, limma version 3.22.7). Because the experiment has a $2 \times 2$ factorial design with two mouse genotypes and two treatment states, linear models were generated with all 4 groups as factors. Subsequently, contrasts of interest were extracted using moderated t-tests for the following groups: 1. "KOFGF-KOV" (treatment effect in Memo cKO), 2. "WTFGF-WTV" (treatment effect in floxed control genotype), 3. "KOFGF-WTFGF" (KO genotype effect in FGF23-treated mice), 4. "KOV-WTV" (KO genotype effect in vehicle-treated mice), and 5. "(KOFGFKOV)-(WTFGF-WTV)" for the interaction between genotype and treatment. P-values were adjusted by the Benjamini-Hochberg method, controlling for false discovery rate (FDR) across all 5 contrasts as a correction of multiple testing conditions. All sequencing data are available on National Center for 
Biotechnology Information, Sequencing Reads Archive under the identifier PRJNA672305. Gene ontology enrichment was assessed in GOrilla, accessed on Nov 17 th 2020 (32) using differentially expressed genes sorted by adjusted p-values.

\section{Accession codes}

RNAseq data: NCBI SRA, Accession: PRJNA672305 (openly available)

LC-MS/MS kidney data: proteomeXchange accession PXD022342 (openly available)

LC-MS/MS recombinant protein data: proteomeXchange PXD022382 (available through username: reviewer_pxd022382@ebi.ac.uk Password: vnw1Dyyl)

Raw data: Zenodo.org, DOI: 10.5281/zenodo.4293503 (openly available)

\section{Acknowledgement}

The authors wish to thank Daniel Bardy, Laboratoire Central de Chimie, Lausanne University Hospital for the analysis of mouse serum samples; Craig Pattinson and Daniel Stead, University of Aberdeen for protein identification and Ivan Nalvarte, Karolinska Institutet, Stockholm, for protocol and reagent sharing. The authors thank members of the Proteomics Core Facility of the University of Bern; Sandra Calderon and Leonore Wigger at Genomic Technologies Facility, University of Lausanne for RNAseq data acquisition and analysis. The authors thank Uyen Huynh-Do and Stefan Rudloff, Department of Nephrology and Hypertension, University Hospital Bern for helpful discussions and acknowledge infrastructure support by the Department of Nephrology and Hypertension, University Hospital Bern. OB and MBM's work was supported by the Swiss National Science Foundation through the program NCCRKidney.CH, a patient association grant (AIRG-Suisse) and a Novartis Foundation grant. OB is also supported by Swiss National Science Foundation grant 310030-182312.

\section{Conflicts of interest}

All authors declare that they have no conflict of interest to report.

\section{Author participation}

MBM and $\mathrm{BH}$ conceived the study. KB, SKR, SB, BH, DS, OB and MBM participated in experimental design. KB, SKR, SB, FD, AP, YZ, and MBM performed experiments. KB, SKR, DS, NEH, OB and MBM 
bioRxiv preprint doi: https://doi org/10.1101/2020.12.04 402511; this version posted December $11,2020$. The copyright holder for this preprint (which was not certified by peer review) is the author/funder, who has granted bioRxiv a license to display the preprint in perpetuity. It is made available under aCC-BY-NC-ND 4.0 International license.

participated in data analysis and interpretation. DS and NEH provided crucial laboratory materials. OB and MBM acquired funding for the research. $\mathrm{OB}$ and MBM supervised the research. KB and MBM wrote the manuscript. All authors critically read and commented on the manuscript and agreed to manuscript submission.

\section{References}

1. Zaoui K, Honoré S, Isnardon D, Braguer D, Badache A. Memo-RhoA-mDia1 signaling controls microtubules, the actin network, and adhesion site formation in migrating cells. J Cell Biol. 2008 Nov 3;183(3):401-8.

2. Marone R, Hess D, Dankort D, Muller WJ, Hynes NE, Badache A. Memo mediates ErbB2-driven cell motility. Nat Cell Biol. 2004 Jun;6(6):515-22.

3. Schotanus MD, Van Otterloo E. Finding MEMO-Emerging Evidence for MEMO1's Function in Development and Disease. Genes. 2020 Nov 6;11(11).

4. Jiang K, Yang Z, Cheng L, Wang S, Ning K, Zhou L, et al. Mediator of ERBB2-driven cell motility (MEMO) promotes extranuclear estrogen receptor signaling involving the growth factor receptors IGF1R and ERBB2. J Biol Chem. 2013 Aug 23;288(34):24590-9.

5. Haenzi B, Bonny O, Masson R, Lienhard S, Dey JH, Kuro-o M, et al. Loss of Memo, a novel FGFR regulator, results in reduced lifespan. FASEB J Off Publ Fed Am Soc Exp Biol. 2014 Jan;28(1):327-36.

6. Kuro-o M, Matsumura $\mathrm{Y}$, Aizawa $\mathrm{H}$, Kawaguchi $\mathrm{H}$, Suga $\mathrm{T}$, Utsugi $\mathrm{T}$, et al. Mutation of the mouse klotho gene leads to a syndrome resembling ageing. Nature. 1997 Nov 6;390(6655):45-51.

7. Shimada T, Kakitani M, Yamazaki Y, Hasegawa H, Takeuchi Y, Fujita T, et al. Targeted ablation of Fgf23 demonstrates an essential physiological role of FGF23 in phosphate and vitamin D metabolism. J Clin Invest. 2004 Feb;113(4):561-8.

8. Moor MB, Ramakrishnan SK, Legrand F, Dolder S, Siegrist M, Durussel F, et al. RedoxDependent Bone Alkaline Phosphatase Dysfunction Drives Part of the Complex Bone Phenotype in Mice Deficient for Memo1. JBMR Plus. 2018 Jul;2(4):195-205.

9. Moor MB, Ramakrishnan SK, Legrand F, Bachtler M, Koesters R, Hynes NE, et al. Elevated serum magnesium lowers calcification propensity in Memo1-deficient mice. PloS One. 2020;15(7):e0236361.

10. Moor MB, Haenzi B, Legrand F, Koesters R, Hynes NE, Bonny O. Renal Memo1 Differentially Regulates the Expression of Vitamin D-Dependent Distal Renal Tubular Calcium Transporters. Front Physiol. 2018;9:874.

11. Gurda GT, Crozier SJ, Ji B, Ernst SA, Logsdon CD, Rothermel BA, et al. Regulator of calcineurin 1 controls growth plasticity of adult pancreas. Gastroenterology. 2010 Aug;139(2):609-19, 619.e1-6.

12. Qiu C, Lienhard S, Hynes NE, Badache A, Leahy DJ. Memo is homologous to nonheme iron dioxygenases and binds an ErbB2-derived phosphopeptide in its vestigial active site. J Biol Chem. 2008 Feb 1;283(5):2734-40. 
13. Miki $\mathrm{H}$, Funato $\mathrm{Y}$. Regulation of intracellular signalling through cysteine oxidation by reactive oxygen species. J Biochem (Tokyo). 2012 Mar;151(3):255-61.

14. Truong TH, Carroll KS. Redox regulation of epidermal growth factor receptor signaling through cysteine oxidation. Biochemistry. 2012 Dec 18;51(50):9954-65.

15. Wright VP, Reiser PJ, Clanton TL. Redox modulation of global phosphatase activity and protein phosphorylation in intact skeletal muscle. J Physiol. 2009 Dec 1;587(Pt 23):5767-81.

16. Rusnak F, Reiter T. Sensing electrons: protein phosphatase redox regulation. Trends Biochem Sci. 2000 Nov;25(11):527-9.

17. MacDonald G, Nalvarte I, Smirnova T, Vecchi M, Aceto N, Dolemeyer A, et al. Memo is a copper-dependent redox protein with an essential role in migration and metastasis. Sci Signal. 2014 Jun 10;7(329):ra56.

18. Ewald CY, Hourihan JM, Bland MS, Obieglo C, Katic I, Moronetti Mazzeo LE, et al. NADPH oxidase-mediated redox signaling promotes oxidative stress resistance and longevity through memo-1 in C. elegans. eLife. 2017 13;6.

19. Gee HY, Saisawat $P$, Ashraf $S$, Hurd TW, Vega-Warner $V$, Fang $H$, et al. ARHGDIA mutations cause nephrotic syndrome via defective RHO GTPase signaling. J Clin Invest. 2013 Aug;123(8):3243-53.

20. Auguste D, Maier M, Baldwin C, Aoudjit L, Robins R, Gupta IR, et al. Disease-causing mutations of RhoGDla induce Rac1 hyperactivation in podocytes. Small GTPases. 2016 02;7(2):107-21.

21. Behring JB, van der Post S, Mooradian AD, Egan MJ, Zimmerman MI, Clements JL, et al. Spatial and temporal alterations in protein structure by EGF regulate cryptic cysteine oxidation. Sci Signal. 2020 21;13(615).

22. Kim J-G, Kwon H-J, Wu G, Park Y, Lee J-Y, Kim J, et al. RhoA GTPase oxidation stimulates cell proliferation via nuclear factor-KB activation. Free Radic Biol Med. 2017;103:57-68.

23. Shin E-Y, Lee C-S, Cho TG, Kim YG, Song S, Juhnn Y-S, et al. betaPak-interacting exchange factor-mediated Rac1 activation requires smgGDS guanine nucleotide exchange factor in basic fibroblast growth factor-induced neurite outgrowth. J Biol Chem. 2006 Nov 24;281(47):3595464.

24. Barrios J, Wieder R. Dual FGF-2 and intergrin alpha5beta1 signaling mediate GRAF-induced RhoA inactivation in a model of breast cancer dormancy. Cancer Microenviron Off $\mathrm{J}$ Int Cancer Microenviron Soc. 2009 Dec;2(1):33-47.

25. Flinder LI, Timofeeva OA, Rosseland CM, Wierød L, Huitfeldt HS, Skarpen E. EGF-induced ERK-activation downstream of FAK requires rac1-NADPH oxidase. J Cell Physiol. 2011 Sep;226(9):2267-78.

26. Lamaze C, Chuang TH, Terlecky LJ, Bokoch GM, Schmid SL. Regulation of receptor-mediated endocytosis by Rho and Rac. Nature. 1996 Jul 11;382(6587):177-9.

27. Grassart A, Dujeancourt A, Lazarow PB, Dautry-Varsat A, Sauvonnet N. Clathrin-independent endocytosis used by the IL-2 receptor is regulated by Rac1, Pak1 and Pak2. EMBO Rep. 2008 Apr;9(4):356-62.

28. Bryant DM, Wylie FG, Stow JL. Regulation of endocytosis, nuclear translocation, and signaling of fibroblast growth factor receptor 1 by E-cadherin. Mol Biol Cell. 2005 Jan;16(1):14-23.

29. Reider A, Wendland B. Endocytic adaptors--social networking at the plasma membrane. J Cell Sci. 2011 May 15;124(Pt 10):1613-22. 
bioRxiv preprint doi: https://doi org/10.1101/2020.12.04.402511; this version posted December 11, 2020. The copyright holder for this preprint (which was not certified by peer review) is the author/funder, who has granted bioRxiv a license to display the preprint in perpetuity. It is made available under aCC-BY-NC-ND 4.0 International license.

30. Hayashi S, McMahon AP. Efficient recombination in diverse tissues by a tamoxifen-inducible form of Cre: a tool for temporally regulated gene activation/inactivation in the mouse. Dev Biol. 2002 Apr 15;244(2):305-18.

31. Gattineni J, Alphonse P, Zhang Q, Mathews N, Bates CM, Baum M. Regulation of renal phosphate transport by FGF23 is mediated by FGFR1 and FGFR4. Am J Physiol Renal Physiol. 2014 Feb 1;306(3):F351-358

32. Eden E, Navon R, Steinfeld I, Lipson D, Yakhini Z. GOrilla: a tool for discovery and visualization of enriched GO terms in ranked gene lists. BMC Bioinformatics. 2009 Feb 3;10:48. 